révolution suivante, on retrouve une situation identique à l'extrémité orientale de la Sibérie.

Cette orbite permet à Arktika-M1 de réaliser ses observations pendant deux périodes de dix heures, chacune étant centrée sur midi solaire (12 h TSM). Cela donne donc 20 heures d'observation dans de bonnes conditions sur une journée.

Le service météorologique russe a diffusé la première image obtenue par
Arktika-M1 (figure 2). Elle donne la même impression qu'une vue de satellite géostationnaire, l'altitude est à peu près la même.

En connaissant l'instant précis de la prise de vue, le logiciel Ixion calcule la position et l'altitude du satellite à partir des données Norad (Capderou, 2017a). On peut restituer les conditions exactes de vue (figure 3 ).

L'intérêt de l'orbite Arktika apparaît clairement sur les figures 1, 2 et 3 . La vue sur la zone polaire nord est quasi permanente (20 heures sur 24). La même orbite exactement, avec seulement un changement de l'argument du périgée $\left(90^{\circ}\right.$ au lieu de $\left.270^{\circ}\right)$, permettrait d'avoir le même point de vue sur la zone polaire sud. Mais qui va financer Antarktika? Laboratoire de météorologie dynamique, Sorbonne Université / CNRS / École polytechnique, Palaiseau

\title{
Ouranos AuRA : accompagner les territoires dans leur transition climatique
}

La structure Ouranos AuRA, collectif de chercheurs de la région AuvergneRhône-Alpes principalement centré sur le pôle universitaire grenoblois, a pour objectif de faire le lien entre science et société sur la question du changement climatique et de ses impacts sur les territoires, particulièrement en montagne. Les collectivités des Alpes du Nord sont en effet particulièrement impactées par les premiers effets du réchauffement dans leurs activités agricoles ou forestières et dans l'industrie des sports de montagne et du tourisme, du fait, entre autres, d'un moindre enneigement et du recul glaciaire. Elles doivent aussi faire face à une recrudescence de phénomènes naturels extrêmes qui viennent régulièrement perturber les réseaux de transport ou parfois même frapper les populations. Les acteurs de ces collectivités sont incités par la réglementation à prendre en charge ces effets et à mettre en place des mesures pour y faire face localement, tant pour réduire les causes du réchauffement en jouant sur des mesures d'atténuation qu'en affrontant les effets immédiats au travers des stratégies d'adaptation. Cependant, si les outils de planification existent (les Plans Climat Air Énergie Territoriaux, PCAET, par exemple), ils restent souvent assez flous sur la mise en œuvre de mesures réellement concrètes sur le terrain. Des connaissances font défaut sur leurs répercussions à différentes échelles de temps et d'espace, alors qu'elles permettraient de mieux anticiper l'avenir des territoires. Mais, au-delà de ces connaissances, l'approche même des nouvelles formes de développement sont à réinventer dans un contexte de changement climatique et plus largement de changement global. Si les acteurs des territoires ont conscience de cette nécessité, les méthodes pour leurs mises en œuvre et pour accompagner ces changements leur font souvent défaut.

La communauté académique grenobloise est depuis plusieurs décennies investie dans l'étude du changement climatique et de ses impacts sur différents types de territoires et notamment sur les Alpes. Au-delà de l'implication de plusieurs scientifiques dans le Giec et l'IPBES, nombre d'entre eux, de différentes disciplines, sont également engagés dans des études plus locales, sur l'observation des évolutions climatiques, de leurs effets sur les glaciers, le pergélisol, les espèces végétales et animales, et les dynamiques territoriales. Ces travaux se font dans un cadre partenarial, plus ou moins soutenu, avec les acteurs locaux. Mais, devant les questions et défis qui se posent aujourd'hui pour les territoires, les demandes de collaboration se renforcent.

Ouranos AuRA a ainsi été créé en 2012 au sein du groupement d'intérêt scientifique (GIS) Envirhônalp, qui visait notamment à faciliter les interactions entre scientifiques et acteurs des territoires rhônalpins sur les questions environnementales au travers du développement de plateformes d'interface. Ouranos AuRA est donc l'une de ces plateformes, centrée sur le climat. Elle doit son nom au consortium Ouranos québécois, développé au début des années 2000, qui a vu dans notre demande une opportunité de déployer son modèle à l'international. L'idée de départ était d'une part de mettre à disposition les connaissances scientifiques développées dans les différents établissements académiques de la région Rhône-Alpes (devenue depuis Auvergne Rhône-Alpes) sur la question climatique et d'autre part d'identifier, dans les questions posées par les acteurs des territoires, les potentiels germes de problématiques de recherche à développer dans un partenariat science-société pour créer de nouvelles connaissances, utiles aux acteurs locaux. Depuis 2018 et la fin du GIS, les engagements d'Ouranos AuRA se poursuivent à l'échelle de l'ensemble de la nouvelle région Auvergne Rhône-Alpes, avec l'appui de la communauté académique du pôle grenoblois, très impliquée sur cette question, et de l'université GrenobleAlpes particulièrement. Plusieurs projets de recherche sont venus alimenter ces activités. Les derniers en date sont les projets Trajectories (financement 
Idex grenoblois) sur les trajectoires des socio-écosystèmes de montagne (2017-2020), Artaclim sur l'adaptation des territoires de montagne (20172020), Sur une coquille de noix (SUN, financements multiples) qui explore avec un collectif d'artistes les perspectives possibles qu'ouvre l'art dans la médiation des connaissances scientifiques sur le climat auprès de différents publics.

Ouranos AuRA a par ailleurs facilité la mise en lien entre des chercheurs associés au collectif et des collectivités pour développer des projets partenariaux en cours actuellement. Ainsi, le projet Climat Métro est un partenariat entre Grenoble Alpes Métropole et l'université Grenoble-Alpes sur la question de la résilience de la métropole grenobloise face à l'évolution des risques générés par le changement climatique, notamment les inondations. Le projet Index (financement Région Auvergne Rhône-Alpes) vient compléter Climat Métro, en ciblant spécifiquement la résilience des activités industrielles face aux risques de crues sur la région grenobloise. Le projet Amorce (financement Région Auvergne-Rhône-Alpes) associe des chercheurs grenoblois et clermontois avec les collectivités du HautChablais et du Sancy pour réfléchir à la diversification touristique comme modalités d'adaptation pour les territoires de montagne.

Ouranos AuRA a également pour mission d'organiser des temps d'échanges entre chercheurs et acteurs des territoires, afin de faciliter les interactions et de rendre compte de l'avancée de certaines recherches. À ce titre et sous le patronage du Comité national français des changements globaux (CNFCG), Ouranos AuRA a organisé le $3^{\text {e }}$ colloque national Action Climat intitulé « Adaptation, atténuation, actions climatiques dans les territoires » du 25 janvier au 3 février 2021 (colloque numérique, cf. figure). Cet événement a permis de rassembler 234 participants, mêlant à parts égales des chercheurs d'un côté et des représentants de collectivités, d'entreprises, d'associations citoyennes ou de la société civile de l'autre. Outre les quatre champs thématiques abordés (agriculture et forêt ; eau et tourisme ; infrastructures de mobilité ;

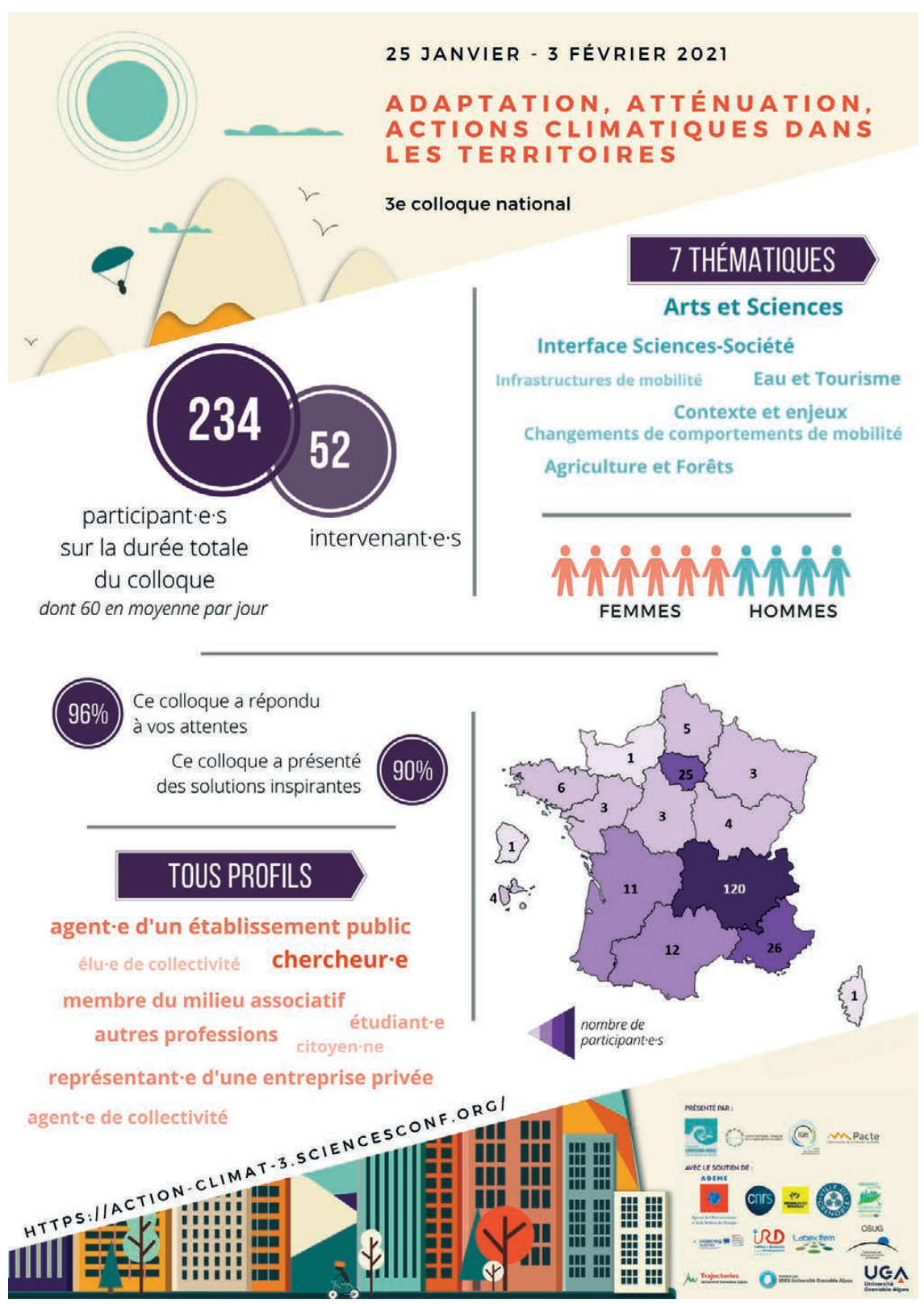

changements de comportements), les approches intégrant arts, sciences et société ont par ailleurs été mises à l'honneur autour de deux sessions qui étaient également ouvertes au grand public. Dans la continuité du $2^{\mathrm{e}}$ colloque national de Marseille en 2018, celui-ci a mis l'accent sur les solutions pour les territoires. Il ressort des discussions que la différenciation utile pour les scientifiques, entre atténuation et adaptation, n'a pas forcément de sens pour les acteurs locaux, dont les actions entremêlent souvent les deux volets. Il apparaît également, sur les quatre champs thématiques abordées, une nécessité de réorganiser l'approche en brisant la logique de silo, tant du côté de la recherche que des acteurs des territoires. Cela révèle un besoin fort d'accompagnement, tant des chercheurs que des acteurs locaux, en termes d'inter et de transdisciplinarité, démarches qui constituent l'essence même du collectif Ouranos promouvoir dans ses activités à venir.

Céline Lutoff

Pacte Laboratoire de sciences sociales, Grenoble

Sandrine Anquetin, Marie Arthuis

Institut des Géosciences de l'Environnement, Grenoble AuRA et qu'il souhaite continuer de 